20

\title{
Shenzhen Net Based on ATM and Internet Techniques
}

\author{
Li Zhou, Gang Wu, Gaohua Wang \\ Shenzhen Statistic and Information Bureau \\ 1 Tongxin Rd. \\ Shenzhen, 518027 \\ China \\ Tel: $+86-755-224-3059,224-1290$ \\ Fax: +86-755-224-1559 \\ E-mail:wang@sz.gov.cn
}

\begin{abstract}
This thesis details the key technologies applied to Shenzhen Net and the multimedia service provided. As an important part of the Project of Shenzhen information construction plan, Shenzhen Net is an Metropolitan Area Network (MAN) covering the whole city based on ATM and Internet technology, it provides government agencies and citizens with comprehensive multimedia information service.
\end{abstract}

Keywords

ATM, Internet, broad-band, multimedia, IS

\section{INTRODUCTION}

Information Systems in the WWW Environment C. Rolland, Y. Chen \& M. Fang (Eds.)

(C) 1998 IFIP. Published by Chapman \& Hall 
The famous former leader of China -- Mr. Deng Xiaoping had ever said in 1984: "Develop information resources to serve China's modernization". While Mr. Jiang Zemin, Secretary of the Central Committee of Chinese Communist Party emphasized that "none of the four modernization (modernization of agriculture, industry, national defense and science \& technology) can be realized without information technology". Their words are regarded as direction of China's IT development. The deployment of a regional information center has been put into "The Ninth Five-year Plan of Shenzhen National Economy and Social Development" as an important part, which indicates that IT development is not only a fundamental national policy, but also an urgent need for China's further economic and social advance. To successfully construct Shenzhen Net, we've got to overcome a lot of technological problems, try to support national IT industry and exploit information resources, so as to drive Shenzhen's IT development to an International level and build the city into a regional information centre.

We adapt mature hardware and software available (router, exchange equipment, storing equipment, etc.) to build this network. Design of the hardware deployment is up to technologically high standard, which focus on MAN (Metropolitan Area Network) multimedia coordination. This design use internationally recognized industry standard, demanding easy transplant of system software and application software as well as simple expansion and replacement of networking hardware and server hardware, thus realizing the feature of opening and general purpose. In addition to applying Latest design tools and test tools, we also work through international cooperation to shorten the time for network building and application system developing as much as possible.

We plan and build the network at the same time with the whole project divided into 3 stages. In the first stage, we built two central nodes and Internet Exits for the OA Service System for Municipal Government Leaders. The second stage is planed to build the ATM Backbone Service System for the five districts of Shenzhen City, and construct key application systems. In the third stage, we will build sub-network for all government agencies as well as standard interfaces for enterprises to access to the network, hence realize "every entity has its own LAN and web site, every LAN going through the same information high-way, every user has his own E-mail address". The whole system will take 4 years to conclude. Investment to this network would amount to 280 million RMB till 1999 when the project is completed. Presently, The first stage is over and the rendered system has been put into use. We are now working on the second stage and look forward to finish the whole project in 1999. 


\section{STRUCTURE OF THE SYSTEM}

According to the standards of OSI, Shenzhen Net can be divided into physical transport layer, network layer, service layer and application layer in terms of hierarchical structure. The illustrations and charts are as follows (figure 1):

Shenzhen Net's Layer Model

\begin{tabular}{|l|l|}
\hline Application Layer & $\begin{array}{l}\text { Municipal leader information } \\
\text { services system, Social information } \\
\text { service system, Database etc. }\end{array}$ \\
\hline Services Layer & E-mail, WWW, VOD, VC,VB \\
\hline Network Layer & Broadband intranet network \\
\hline Physical Layer & $\begin{array}{l}\text { ATM skeletal, public transport } \\
\text { system }\end{array}$ \\
\hline
\end{tabular}

Figure 1 The hierarchical structure of the system

The system mainly includes three parts: the kernel part, the peripheral part and the interconnecting part. Their contents are as follows:

(A) The kernel part refers to the subsystem made up of two central nodes of the city's information office buildings A and B, and the first, second offices of municipal government affairs, the People's Congress, the People's political consultative conference and the skeletal nodes of the five districts in Shenzhen city. Which support the service system for the municipal leaders to handle official business, macroscopic decision-making supporting system and the five leading organization's automation systems to handle official business, including also the firewall system, data and network management and charging system.

(B)The peripheral part refers to the network of automation systems to handle official business of the 88 departments(bureau level), and also includes intranet and application systems of thousands of state-owned enterprises. These application nodes are not only the service objects but also the resource centers to serve the society and feedback the government.

(C) The public interconnecting part refers to the exchanging devices, circuits and application software to be connected to the kernel and the peripheral ones. It also includes such nodes as Golden-bridge network, Science and technology network, CEINet (Chinese Economic Information Network), 
as well as the interconnection with the local ISP(i.e. the post and telecommunication network, campus network etc.)

\section{THE NETWORK'S PHYSICAL STRUCTURE}

The system's physical structure is composed of seven 622M ATM skeletal nodes. The skeletal ATM exchangers of the five Districts are connected with ones of the city's information office buildings A and B via optical fiber cable, and the networks of the municipal Five Leading Organizations are connected with each other with optical fibers. We also reserve 2-4 pairs of standby replacement redundancy and optical fibers to extend the capacity. When it come to the constructions of the skeletal system, we inter-connected them with STM-4(622Mb) interfaces to form a full connection, so as to ensure the security of the network, adequate capacity, consequently facilitating the maintenance, dispatching and managing network.

The network center is configured with two sets of skeletal ATM exchangers, with each is configured with sixteen OC3 ports and double power supply systems to form redundancy configuration. Apart from providing tolerant ability, it also bear the ever-increasing network loads. Each of the two sets of exchangers of the network center is configured with seven pieces of STM-4 module to link with the five districts' exchangers, as well as with two pieces of STM-1/OC-3 module to link with the network center's main ATM exchangers, each is also configured with 8 ports E1/E3 ATM, frame relay card and four pieces of $\mathrm{CE}$ cards to facilitate other bureau and commissions under the municipal government to set up connection network. Every department has an ATM skeletal exchanger, other districts' ATM exchangers are configured with STM-4, STM-1/OC-3 ATM modules and some fixed 8 ports E1/E3 ATM, frame relay card and four pieces of CE cards to facilitate other districts' sub-bureau to set up network-connecting.

The kernel broadband network attachment is a broadband high-speed local area network(LAN), which supply services for the five leading organization leaders to handle official business and make decisions. It is connected to the leader's desk-top directly through ATM desk-top exchanger of $25 \mathrm{Mbps}$ and inserted into the skeletal network through OC-3/STM$1(155 \mathrm{Mbps})$. The main devices of the kernel broadband network attachment include server, multimedia PC,ATM exchangers, Ethernet exchangers, mainstay routers etc., so as to satisfy the needs of supplying information for the municipal leaders to handle official business and make decisions through multimedia,(such as video-meeting, VOD, remote multimedia emergency commands, official document exchanging etc.)

The bureau and commissions under the second office are inserted into the network central skeletal exchangers through the second office's ATM mainstay exchanger via OC-3/STM-1 optical fiber cable. Several other offices 
such as Public Security Bureau, Finance Bureau, Party school, Reception Office, The Three Defence Office. Port Office etc., adopt optical fiber to be inserted into the skeletal network. The main devices of these units are composed of servers, multimedia PC, ATM mainstay exchangers, ATM desktop exchangers, Ethernet exchangers, routers etc., in order to satisfy each units' needs to multimedia office information services, for example, video meeting, VOD, official document exchanging, the reporting to the leadership and creating of the multimedia information, E-mail, database inquiring and other applications.

The inserted modes to support the rest bureau and commission offices are: HFC, Frame relay and N-ISDN, DDN, PSTN as well as $2.4 \mathrm{G}$ wireless extending frequency link, Among which, HFC, 2.4G extended frequency link are the main inserted modes, and N-ISDN, PSTN are reserved modes. When HFC and frame relay have faults, the network will put N-IS DN,PSTN through automatically.

The illustrations of the general construction of the physical structure of the skeletal networks are as follows: 


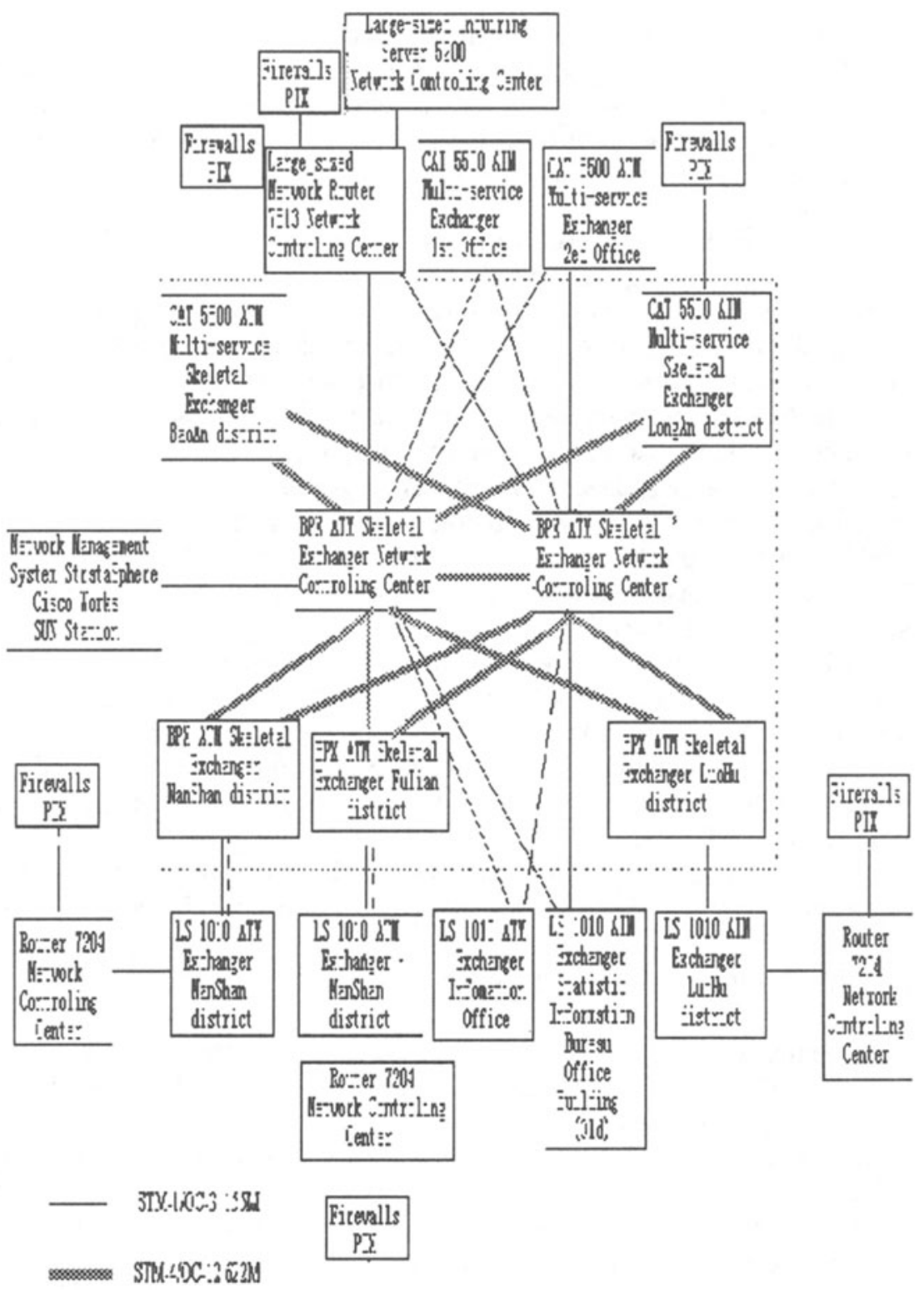

Figure 2 physical structure of Shenzhen Net

4 NETWORK MANAGEMENT SYSTEM 
* the functions of network management system

The main functions of network management system includes: network's fault management, network's configuration management, network's performance management, network node facilities management, network security management, traffic flow accounting and charging.

* the components of network management system

Besides controlling the transport layer and network layer, the network management system also provides application management ,such as automatic backup of various important data, VOD user management ,service charging management, the replacement and maintenance of programs.

The NMS is composed of two parts :the management of network basic facilities and the network management of application layer .The former controls the transport layer's devices and circuits, for example , ATM exchangers, routers , servers, Ethernet exchangers, device configuration ,faults, network topology maintenance, virtual circuit traffic and congestion . This layer is to mainly control and maintain the services, charging and accounting of users of application system.

NMS adopts HP-Open View as network management platform ,Sun advanced work station as NM server and NMS as network management software which is provided by ATM backbone network services supplier.

NMS is developed on the basis of special application and Database system and use the ratified servers of Netscape company to realize Internet / Intranet' according management.

Network analyzer Sniffer, fabric cable tester, wire cable tester and other testing and analyzing equipment are used in controlling communication links.

\section{MAIN BUSINESS SYSTEMS}

The creation of basic ATM backbone network meets the needs of varied kinds of information of articulation, data and pictures. The multimedia services supplied by Shenzhen Net are:

* video meeting service: holding meetings in the meeting rooms of the first office building of municipal government, the Standing Committee of the National People's Congress, district committee of five districts and the standing committee of the government by using TV, which makes one side see the scene of the other side as well as hear their voice,just like having a meeting at the same place and communicating face-to-face. Now the five leading organizations have put the desk visual meeting into practice and gained much convenience from it.

* virtual private network : Setting up local network for users of offices of bureau level by using broadband network to provide high speed data channel, 
which has a high data transmission speed and can transmit data ,pictures, articulation with fault-tolerant functions.

* VOD: the video service on the Shenzhen Net, which allows the users of five leading organizations to see the TV programs they want at any convenient time.

\section{INTERNET/ INTRANET APPLICATIONS}

\subsection{NETWORKING PROTOCOL}

The network supports widely-used TCP/IP protocols ,IPX/SPX protocols and the SNA, DECnet

commenly - used in host system.

\subsection{SUBNET PARTITION AND IP ASIGNMENT}

Shenzhen Net have two layer, the one is the Internet layer, the two is the internal network specially used by the government and a MAN in Shenzhen. So it adapts two sets of IP address, that is, legal Internet IP address and internal IP address. Up to now we have applied for 32 IP addresses of $C$ class and used them for Internet services. Internal IP address is used on internal network, which on one hand can avoid the problem of the lack of address resources on the Internet, on the other hand can strengthen the security protection and control of all kinds of information resources on special network. In practice, we use a Internal IP address of A class(10.0.0.0) as the address source of the whole government's special network and give a unified distribution and management to address on this base. To control it efficiently, we divide the network into several subnet and make them the composed units of the government's special network.

\subsection{DOMAIN NAME ALLOCATION}

Shenzhen Net's three-grade domain name registered in China Internet network information center is .sz.gov.cn. The general domain name server in network centre gives a unified control to the allocation and use method of domain name in the whole Shenzhen Net. To realize an easy management and avoid too long sub-domain name, we connect Internet domain name with internal network domain name. Attach .gov.cn to internal network domain name and you will get Internet domain name. In internal network domain name the abbreviation for Shenzhen, sz. is taken as the top grade of domain name, skeletal nodes of the five districts as the second grade domain name, that is, $\mathrm{ft}($ Futian district), lu(Luohu district), nh(Nanshan district), ba(Bao'an district), lg(Longgang district), the concerning governmental 
department and enterprises of each district as the third grade domain name . Those concerning governmental department and enterprises which in the charge of municipal administration have the right to allocate the second grade domain name directly, stood by three alphabets to distinguish from the second grade domain name of the five districts. And all of the above form a complete domain name system.

\subsection{NETWORK SECURITY}

It can be divided into security protection inside the government's special network and for the insertion of the government's special network and external network. And the former is realized by setting up virtual network(VLAN) to create different subnets relatively independent, inside which the resources are free access and use, but between which it needs a strict security access control to communicate with each other. Then we can ensure the whole security of network with the control from network layer to application layer.

Apart from applying intensive security control in the network center, on the insertion of each subnet, we can take additional security measures, like setting up special firewall and protecting internal network strictly.

When subnets are communicating with each other across special network, a comparably mature technology ----tunnel technology is used to set up a virtual network for a department or an enterprise, which made the efficient communication of information resources available on the premise of ensuring security.

The later takes many protective measures, at the interface: fixing filter on insertion router; using firewall Cisco PIX; using proxy; using different communication protocols in-and outside, the host at the interface supporting two kinds of protocols. In the data transmission : application layer's data encryption (using the network security encryption product appointed by the national security code management committee); virtual network technology.

\subsection{ISP NETWORK INTERCONNECTION IN SHENZHEN CITY}

Equipping the network center with a set of large scale router system with dual redundant backup and adopting static route technology to make the ISP netwoek in Shenzhen city inter-connect and inter-communicate with security buffer . Each service network can be attached to network centre through Shenzhen Net. The inter-connected networks include: cable television HFC network, campus network of Shenzhen University, Shenzhen node in ChinaNet, Shenzhen Wanyong network, China online, InfoHiway network and so on. 


\subsection{EXTRA INTER-CONNECTION}

Shenzhen Net inter-connects with other places in and out of China through three Channels:

- using of 2M satellite network to connect with CSTNet, CNNIC and CERNet, this channel is used by concerning governmental departments.

- connecting CHINAGBN through 64K satellite network, which mainly acts as a Internet exit to society service and a backup one to government service.

- connecting CEINet (China Economy Information Network) through $64 \mathrm{KDDN}+64 \mathrm{KX} .25$, two leased lines, which can communicate with all the information centres in every province or city throughout the country.

\section{APPLICATION SYSTEMS}

There are three layers in the application system: the decision support system, the comprehensive system and the professional system. The decision support system is the highest layer in the application system and serve the five leading organizations directly; The comprehensive system is in the middle of the application system. It serves every department of the government and only serve one or several special bureau offices or district government.

\section{- The decision support layer application}

The decision support layer application system, which is oriented to the five leading organizations, is the highest layer in the application system of Shenzhen Net. It can also be divided into three parts: the municipal leader office service system, the macro decision support system and the municipal five leading organizations office automation system.

The municipal leader office service system can provide many kinds of information for the municipal five leading organizations, including various multimedia information such as text, pictures, video, sound and so on.. Especially, the real-time dynamic economic information and video information can provide the leaders decision service by pushing information to the front of the leaders through the leaders desk-top PC. These is mainly embodies in providing reference information for leader decision ,exchanging and passing the decision opinions made by leaders through network and so on. It supply seven kinds of service:
1. Information inquiry services;
2. VOD services;
3. Video conference services;
4. Dynamic video monitor information service;
5. Real-time stock information services;
6. Internet services(www, e-mail, newsgroup etc.); 
7. cable television services.

Macro decision support system, which is based on the analysis and predetermination of macroeconomics and social development, can provide the leader decision reference by the construction of model base, method base and knowledge base.

Municipal five leading organizations office automation system, which is the base of management decision conduct information on decision layer, is to realize the electronical of administrative body's everyday business and to realize the exchange of electronic document. This system will not only ameliorate the procedure of handling affairs, but also realize the sharing of information and the office without paper by this procedure. It will greatly promote working efficiency of the office staff, strengthen cooperation and communication, raise the efficiency and quality of service for sociaty, advance constructions of office-style and integrity, and promote the image of government .

\section{- Comprehensive layer application}

Comprehensive layer application system including statistics information system, social public information service system, government information service system, gold seacoast information service system, Shenzhen Internet Institute, Shenzhen Net navigator and so on.

\section{* Shenzhen Net navigator and full text search}

Though each government departments' network constructed information resource databases independently, these information resource databases are scattered and need a unified administration. This system integrate all information resource to formulate the information service total catalogue of whole city and provide the function of full text search. Because of the impossibility to integrate each professional network information physically, the resolution is to construct these scattered information source into a large scale distributed database. Shenzhen Net navigator is the catalogue service system of Shenzhen Net which can insure the good information orderliness and high search efficiency of Shenzhen Net.

\section{* Statistics information system}

Statistics information system is based on the supply of authoritative statistics data, facing every levels from leaders to public and reflecting the allround general situation of social economic development. This system takes statistics index as keyword, and database as kernel, breaking tradition report structure and adopting Oracle8.0.The part of statistics data collection adopts Internet browser technology, and the data process part adopts the waterfall model and prototype method with component theory to set up the universal 
component data base and the special ones which makes it possible for users to self-define every kind of combination of inquiry mode and output mode.

\section{* Government information service system}

Government information service system is a window in Internet to publish municipal government information, each district government and other bureau. It circulates the information made by ShenZhen government to the public simultaneously by taking advantage of the public network platform of Shenzhen Net and this will greatly help the municipal government open working procedure to public and improve work efficiency. In this system , users can acquire the organization arrangement of each government, the function introduction and the policy regulation. Besides, it is also available to inquire important speech of government official and the report of the governmental work.

\section{* Social public information service system}

This system ,which is relied on the database at home or abroad and the associative search, takes advantage of various public communication platforms and information terminals to provide public information search and circulating service at all times and places with a renewal of 3,000,000 words every day. This system adopted Internet technology and was put into use in 1996 with some characterized application software.

\section{* Shenzhen Internet Institute}

Shenzhen Internet Institute is a garden on network for the leaders and cadres of Shenzhen on every level to study modern information technology and concerning administrative knowledge. It is composed of some columns such as administrative library, study on network, discussion on network ,Internet navigator and so on. Bsides, TRS full text search system was adopted and firewall has also been set up.

\section{* Jing-Jiu information service system}

Jing-Jiu information service system is an information corridor to serve the economic construction connecting 9 provinces and cities, 20 districts and 108 counties which are all along the Jing-Jiu railway. The appearance of it makes Shenzhen Net the information circulating base area with the most wildly cover of information source.

The information of every city and county along the Jing-Jiu railway is made up of investment environment, investment policy, items of inviting outside investment, export product information, ISO9000 product ratified information, supply-demand information and statistic information, which can all be circulated in China, Hong Kong and America on Jing-Jiu information 
network, China gold bridge network and China economic information network.

The local computer information network in every city and county along Jing-Jiu railway provide support and cooperation on hardware, software and techniques each other, by which will gradually set up a wide-area network mode to realize the connection of Jing-Jiu information.

To make the supplement between Shenzhen and Hong Kong, Jing-Jiu information network is going to put emphasis on the collection of statistics information, comprehensive information and economic information of Hong Kong, Taiwan and Shenzhen and will develop a global Chinese information guide system in order to provide a better service.

\section{- Professional layer application}

The professional layer application system is the application system of various profession in economic and society such as financial tax management, enterprise production circulation and financial stock information system in economic field and geography information, enviroment monitor, territory resource information system in society field. Personal market management information system runs well.

\section{INFORMATION RESOURCE DATABASES}

Information resource database is the soul of Shenzhen Net. With the information network which is a basic communication platform in physics, it is possible to provide government, enterprise and public information service with good quality by developing some primary application systems and efficient information base.

The information resource base of Shenzhen Net contains three parts according to the service objects as follows:

- the information base serving government functional department as municipal committee and

government, each bureau office and so on;

- the enterprise information base serving business enterprise

- the public information base serving the social public

The construction of the resource base of Shenzhen Net mainly includes:

- information resource centre of the city----the whole city's information navigator and full text

search

- the information database of decisive reference---- serving the five leading organization of the city

- the information database of the five districts 
- the information database of macro-economy (including statistic information database )

- professional database for various professions

- social public information database (Yellow page database, newspapers and magazines' databases,

tour information bases and so on)

\section{APPLICATION EFFECT}

The successful built of the first stage of Shenzhen Net has evoked strong repercussions in all fields of the state. The powerful predominance of the multimedia application based on ATM avoids detours for other local governmental departments to build their own networks. In very short time, these departments built up each own ATM network and application system one by one. Also, the first-stage construction of Shenzhen Net shed guiding light on the design of the state political and public service system, and gave impetus to the state information construction. Many provinces and cities asked for related information for references.

- After visiting Shenzhen Net and referring to the system planning, ICBC (Industrial and Commercial Bank of China) Headquarters, the Department of State Security, Hunan Provincial Government, Ningbo Municipal Government and Wuxi Municipal Government set up each own multimedia application system one after another. The VOD (Video on Demand) system is able to record TV news reports everyday so that leaders can access it whenever there is a need. And this is one of the original ideas of Shenzhen Net.

- Hainan Provincial Statistics Bureau has employed the statistics dataprocessing system and attained an excellent running effect.

- Tianjin Municipal Government has employed a large amount of Dawning servers, which made in China, as its prime servers.

- Henan Provincial Information Center has employed partial software of Shenzhen social information service system, for example, the WWW information generator, which can automatically format the all information files as HTML format and put it on the http server.

- The village of Asian Games in Beijing completed the general planning and the solution design of its campus network after visiting and consulting with Shenzhen Net designers many times.

The construction of Shenzhen Net has received high praises from the leaders of Central Government and the experts from the Information Office of the State Council. During the year after the complete of the first stage construction, we have entertained over 2000 leaders and experts from all over the world, including those from Germany, Australia and the United States. 
On August 8, 1997, the Hi-tech Department of State Science and Technology Commission hosted an evaluation conference against the first stage of project of Shenzhen Net. The assessment team consists of the leaders and experts from State Science and Technology Commission, Ministry of Electronics Industry, Academy of Sciences of China and Academy of Engineering of China. The final expertise is that the system is of advanced technology, original design, stable running and excellent function, which should be promoted and treated as a successful experience.

Shenzhen Net is the first broadband Metropolitan Area Network in China, which has matched the advanced international level of the same kind of network in mid 90's. Furthermore it has made a significant breakthrough on the collaboration of multimedia and application using ATM technology, which has pushed the construction of our city information network entering a new era and narrowed the gap in this field between China and the developed countries.

\section{BIOGRAPHY}

Li Zhou, Director of Shenzhen Statistics \& Information Bureau Renmin University of China honored professor

Gang Wu, Director of Shenzhen Network Center, Ph.D.

Gaohua Wang, General Engineer of Shenzhen Network Center 